\title{
Protée
}

\section{De l'influence de la présence itérative sur l'accroissement de la profondeur de perspective. Un retour critique sur l'omniscience narratoriale et sur la restriction de champ du personnage}

\author{
Alain Rabatel
}

Résumé de l'article

L'analyse linguistique de la fréquence itérative (vs singulative) entraîne l'augmentation de la perspective du point de vue du personnage, ou une limitation de la perspective du point de vue du narrateur. La perception linguistique ne se limite pas à une saisie perceptuelle hic et nunc: avec l'itératif, des temporalités diverses sont compactées, favorisant une augmentation du savoir de l'énonciateur, ce qui complexifie l'analyse des sources évidentielles (origine du savoir) dans les récits, relativisant les approches ontologiques de l'omniscience narratoriale et de la restriction de champ actorielle.

Éditeur(s)

Département des arts et lettres - Université du Québec à Chicoutimi

ISSN

0300-3523 (imprimé)

1708-2307 (numérique)

Découvrir la revue

Citer cet article

Rabatel, A. (2000). De l'influence de la présence itérative sur l'accroissement de la profondeur de perspective. Un retour critique sur l'omniscience narratoriale et sur la restriction de champ du personnage. Protée, 28(2), 93-104.

https://doi.org/10.7202/030597ar 


\title{
DE L'INFLUENCE DE LA FRÉQUENCE ITÉRATIVE SUR L'ACCROISSEMENT DE LA PROFONDEUR DE LA PERSPECTIVE
}

\author{
UN RETOUR CRITIQUE SUR L'OMNISCIENCE NARRATORIALE \\ ET SUR LA RESTRICTION DE CHAMP DU PERSONNAGE
}

Alain Rabatel

\begin{abstract}
L'imparfait étant letemps prototypiquedu second plan, il joue un rôle fondamental dans la construction des descriptions, comme dans l'expression du point de vuel. De surcroit, l'imparfait est souvent mis à contribution pour la réalisation de la fréquence itérative. La présence, ici et là, d'un même tiroir temporel invite à une réflexion sur les éventuelles relations entre fréquence itérative et perspective narrative: or ces différents concepts sont rarement articulés2, sans douteen raison dela prégnance de la distinction genettienne entre mode (narratif) et voix (narrative). N otre objectif est de montrer que la profondeur de perspective (qui, dansFigures III, relèvedu mode) est affectée par la fréquence (qui, dans la même œuvre, appartient à la voix): en effet, selon que la perspective s'accompagne de la fréquence itérative, la profondeur de perspective augmente, et elle diminue lorsque la perspective va de pair avec la fréquence singulative. Ce phénomène concerne les scènes comme les pauses, que celles-ci soient focalisées par un personnage (I) ou par le narrateur (II). Cette donnée remet en cause I'approchetraditionnelle des profondeurs de perspective du personnage et du narrateur, artificiellement cantonnées à la restriction de champ du personnage et à l'omniscience du narrateur (III).
\end{abstract}

\section{Rappel de définitions}

\section{et cadre théorique de notre approche}

Précisons, en préambule, un certain nombre de définitions concernant la perspective narrative, le point de vue et la profondeur de perspective.

a) La perspectivenarrative concerne la détermination des deux 3 instances (lenarrateur ou le personnage) par lesquelles les informations diégétiques parviennent au lecteur ${ }^{4}$.

b) Le point de vue, centré sur l'analyse des perceptions représentées dans l'arrière plan du texte, est un des concepts qui rend compte des mécanismes construisant ces perspec- tives. N ous en proposons une approche énonciative, largement étayée par les travaux de D ucrot, ainsi quepar ceux de Banfield, à qui nous sommes redevable du concept de phrase sans parole; qui plus est, son analyse du discours indirect librenous a guidépour l'étude du point devue 5 - désormais PDV -, dans la mesure où le débrayage énonciatif qui y opère est analogue dans les deux cas. $D$ ans le cadre de récits hétérodiégétiques, auquel nous cantonneronsnotreanalyse, le PD V exprime à l'intérieur de la voix narrative, les perceptions et les pensées associées d'un énonciateur (ou focalisateur) distinct du locuteur-narrateur. Tel est le paradoxe du PDV : il rend compte de la subjectivité de certaines perceptions et pensées, dans des énoncés comportant troisième personne, et temps du passé- alorsqu'en principe la subjectivité est couplée avec le syncrétisme traditionnel du je-ici-maintenant. Avec le PDV, on se trouve face à des pensées à peine verbalisées, voire des perceptions qui, à premièrevue, paraissent purement objectives. En effet, al ors quec'est toujourslenarrateur qui raconte, certaines perceptions ou certaines pensées renvoient au personnage, quand bien même ce dernier, littéralement parlant, ne dit rien 6 :

(1) II ralluma sa lampe et fit une rapide inspection du blockhaus. Tout paraissait en ordre: le canon était pointé sur ses repères de nuit - auprès du fusil-mitrailleur s'empilaient sur une caissette unetrentaine de chargeurs garnis; dans un angle du béton, le tas brillant des cartouches ruisselait en vrac comme si on venait de le basculer d'une brouette. L'idéelui vint de vérifier une dernièrefoisleboyau d'évacuation.

(J. Gracq, U n Balcon en forêt, Paris, J. Corti, 1958, p. 221)

Les procès en caractères romains correspondent non pas à une description assumée par le narrateur, mais à des perceptions et pensées représentées coréférant à un énonciateurfocalisateur distinct du locuteur-narrateur: ainsi, les per- 
ceptions à l'imparfait renvoient à l'examen du lieutenant $G$ range. $C$ hacun decesénoncés descriptifs, dansle deuxième plan 7 , peut être assimilé aux perceptions et aux pensées non verbalisées du focal isateur-sujet du premier plan, à une sorte de commentaire, comme si Grange se parlait à lui-même pour se rassurer: «Bon, tout est en ordre, le canon est bien positionné, etc. ». Q uand bien même le texte mentionne explicitement l'existence de ces inférences (cf. les deux points, la comparaison finale, ainsi que la dernière phrase confirmant l'existence d'un processus réflexif de G range, amorcé précédemment dans le deuxième plan), elles ne sont pas explicitement attribuées au focalisateur: c'est en cesens que Banfield considère detels énoncés commedes «phrases sans parole».

c) La profondeur de perspective concerne la quantité et la nature desinformations véhiculées par un PDV déterminé ${ }^{\text {: }}$ c'est ainsi qu'en (1b) notre réécriture de (1) exemplifie une profondeur de perspectivesupérieureà celle du texte original :

(1b) II ralluma sa lampe et fit une rapide inspection du blockhaus. T out paraissait en ordre: le canon de 75 était pointé sur ses repères de nuit, exactement comme 0 livon l'avait positionné, d'après les instructions du capitaine Varin - auprès du fusil-mitrailleur de Gourcuff s'empilaient sur une caissette une trentaine de chargeurs garnis; dans un angle du béton, le tas brillant des cartouches ruissel ait en vrac comme si on venait de le basculer d'une brouette. L'idée lui vint de vérifier une dernière fois le boyau d'évacuation.

En (1b), le focalisateur est le même qu'en (1), mais la masse informationnelle transmise au lecteur y est plus importante, et, en ce sens, la perspective de Grange est plus «large» (quantitativement), plus «profonde» (qualitativement: elle témoigne d'un savoir qui déborde ce qu'un observateur lambda verrait s'il était à la place du lieutenant G range) : ces métaphores de la largeur de l'empan perceptif et de la profondeur du volume du savoir indiquent que le focalisateur a accès à des données dépassant le cadre de ce qu'il perçoit dans l'espace et le temps où il rend compte de ses perceptions (parce qu'il a appris telle donnée auparavant, par observation ou par «emprunt à autrui », selon la formule habituelle en matière d'analyse des sources évidentielles 9 ). $\mathrm{Ce}$ sont ces informations quantitatives et qualitatives qui déterminent ici l'accroissement de la profondeur de perspective. Ces concepts étant rapidement illustrés, analysons plus précisément des exemples de PDV, sous l'angle de la fré quence.

\section{Fréquence itérative et point de vue du personnage}

Là où $G$ enetteétablit un tracé rel ativement étanche entre «récit "proprement dit" »10 (sousforme descènes ou de sommaires) d'un côté et description de l'autre, nous proposons de dynamiser ce système. La problématiquedu PD V traverse à la fois des phénomènes relevant du mode et de la voix: en l'occurrence, lesfaits de fréquence concernent à la foisscènes et pauses, plutôt qu'ils ne servent à les discriminer, et ils jouent un rôle notable dans la profondeur de perspective.

Rappelons que sel on $G$ enette, la fréquence se caractérise par trois modal ités d'écriture distinctes: le récit peut raconter unefoisce qui s'est produit une fois (récit singulatif); il peut raconter une fois ce qui s'est produit $n$ fois (récit itératif); il peut enfin raconter $n$ fois ce qui nes'est produit qu'unefois (récit répétitif) (G enette, 1972: 145sqq). Cette fréquence itérative n'a que peu à voir avec l'aspect itératif, même si I'on est tenté de souligner l'homologie entre fréquences itérative et singulative d'une part, et aspects itératif et semelfactif d'autre part. L'opposition aspectuelle entre procès unique et procès répétél1 est d'un autre ordre que la fré quence, qui concerne le rapport entre les évènements de la diégèse et leur narration. Certes, lorsque Zola évoque des combats, dans La D ébâcle, il combine fréquence itérative et aspect itératif; maisil n'y a rien d'automatique, l'aspect itératif n'impliquant pas le choix d'une fréquence itérative, comme le montrel'exemple(1): I'aspect itératif des verbes àl'imparfait s'accommode fort bien d'unefréquencesingulative, dans la mesure où cettescène racontée une fois est censéenes'être produite qu'une fois ${ }^{12}$.

\subsection{Scènesitératives ou singulatives}

La profondeur de perspective de scènes apparemment semblables varie selon la fréquence. Ainsi, les visions «compactées» du personnage-focalisateur avec l'itératif paraissent favorables à l'expression d'une plus grande profondeur de perspective que lorsque les focali isés sont exprimés dans des seconds plans sous la domination fonctionnelled'un premier plan singulatif. Autrement dit, les visions du personnage expriment uneprofondeur de perspectivequi n'est plus nécessairement limitée par le cadre spatial et temporel (désormais $S^{\circ}$ et $\mathrm{T}^{\circ 13}$ ), mais au contraire quasi illimitée, comme le montre l'analyse comparative de (2) et (3):

(2) - Vont se faire torcher, les B oches, proclamait G ourcuff, déjà très rouge et très suant, et devenu optimiste.

Vershuit heures, la laie commença à s'animer. D eux sidecars et une motocyclette passèrent roulant à toute allure vers la frontière. Puis une voiture à fanion, et un détachement du 
génie. D errière la maison forte, du côté des cantonnements de la cavalerie, commença à s'enfler, de plus en plusfort, un ronronnement demoteurs. G range, O livon, G ourcuff et $H$ ervouët s'étaient assis maintenant sur l'appui des fenêtres, les jambes pendantes contrelemur, commeun matin deQ uatorze Juillet. Le soleil flambait dur, la matinéeétait sans nuages. V ers neuf heures, on entendit le vaste ronronnement à l'ouest éclater en pétaradebrutale, pui ss'égaliser et vi rer lentement à unenappe grave, et la cavalerie commença à déboucher.

Ce qui dominait tout, c'était le vacarme; un fracas lourd, térébrant, de tôles, de chaînes, de bidons, de chenilles et de blindages secoués qui prenait à la nuque et ne vous lâchait plus. Les petits groupes de civils au bord de la route - que le défiléfaisait surgir par magie des bois déserts- avaient poussé quelques acclamations au passage des premières voitures, mais ils avaient renoncétrès vite, découragés: ilsattendaient maintenant que finît de défiler ce train de marchandises un peu encombrant; et leshommes, sur les véhicules, passai ent devant eux muets et indifférents, vaguement allégoriques, comme des pompiers assis en rang le long de leurs échelles.

(U n Balcon en forêt, p. 172)

Toute cette description focal isée par l'équipage du fortin (G ourcuff au premier chef, mais aussi ses autres camarades sans compter, à la limite, les focal isateurs additionnels ${ }^{14}$ que forment «les petits groupes de civils») est un second plan descriptif sous la domination fonctionnelle du premier plan singulatif: les visions à l'imparfait développent des pensées, ou des impressions visuelles ou sonores suscitées par des évènements du premier plan. C'est en ce sens qu'il faut comprendre la valeur des procès rapportés à 'imparfait: ainsi, il est certes évident que «le soleil flambait dur», avant même que l'équipage ne soit «sur l'appui des fenêtres, les jambes pendantes», mais le récit est ainsi raconté que ce procès nous est rapporté au moment où il passe par le filtre perceptif des focal isateurs. Aussi, tout ce qui est perçu et pensé est alimenté exclusivement par les évènements narrés dans le premier plan et est étroitement sous la dépendancedu cadrespatio-temporel du premier plan. La perspective dece PDV , sous la domination fonctionnelle d'un premier plan singulatif, réside dans le fait que les informations arrivent au lecteur par la médiation du personnage-focalisateur, et surtout dans le fait que ces informations selimitent àcequ'il perçoit en $\mathrm{S}^{\circ}$ et en $\mathrm{T}^{\circ}$, compte tenu également des inférences qu'il peut effectuer (sur la base de ses perceptions ou du savoir accumulé dans la diégèse). En ce sens, cette scène se caractérise par une profondeur de vision limitée à $\mathrm{S}^{\circ}$ et $\mathrm{T}^{\circ}$, et, de ce fait, limitée par $\mathrm{S}^{\circ}$ et $\mathrm{T}^{\circ}$.
Comparons (3) avec (2): en dépit de profondes similitudes (lePDV y repose dans les deux cas sur la même opposition fonctionnelle des plans, sur la même val eur subjective de l'imparfait renvoyant à la saisie perceptuelle d'un focalisateur particulier ${ }^{15}$ ), ces deux PDV n'expriment pas une identique profondeur de perspective. En effet, (3) offrel'exemple d'une vision quasi illimitée d'une scène, dans la mesure où les informations qui nous proviennent du personnage-focal isateur débordent le cadre des perceptions ou des inférences contenues dans les limites de $\mathrm{S}^{\circ}$ et de $\mathrm{T}^{\circ}$. Et c' cest précisément grâce à l'itératif que cette extension de la profondeur de perspective est obtenue:

(3) Lorsqu'ils revenaient à la cabane, et mangeaient assis côte à côte sur la luge ce qui restait des provisions, l'après-midi savançait déjà; I'horizon desboissefonçait d'un cernemauve. Le froid tombait, et il passait dans la lumière oblique une nuance de tristesse soucieuse. M ona frissonnait sous sa courte veste fourrée: elle s'embrumait tout à coup aussi vite qu'un ciel de montagne, tout entière ouverte aux avertissements de I'heure et de la saison.

- Je n'aime pas les fins de journée, faisait-elle en secouant la tête quand il l'interrogeait. Et quand il lui demandait à quoi elle pensait:

- Jene sais pas. À la mort... Q uel quefois elle roulait la tête sur son épaule, et quelquessecondesellefaisait contrelui sessanglots pressés, si étranges, brusques comme une pluie d'avril. Brutalement, il sentait le froid le saisir. II n'aimait pas les mots qui montai ent à cette bouche de s bylle enfant, soudain pleine de nuit. Q uand ils arrivaient aux Falizes, une ombre bleue et froide coupait lesmursdesmaisonsà mi-hauteur; lesfilaments deglaceregelésdéà au bord desgouttièresemplissaient lesruelles desilence. Avant mêmequelesolél fût couché, la nègedevenait grise. La terre autour d'eux paraissait soudain si éteinte, si glacée, que les pressentiments de $M$ ona gagnaient $G$ range: il sentait la journée basculer d'un coup au fond d'un puits noir, et une eau grise, froide, monter en lui dont il remuait le goût fade dans sa bouche. D ès que Julia avait servi le thé, ils se dévêtaient avec une hâte anxieuse; dans la grande pièce assombrie, toute pleine de la tristesse paysanne du soir, ils sétreignai ent sans parler. Q uelquefois il se redressait à demi contre l'oreiller, entre les draps froids, et, lâchant les doi gts de M ona, lai ssait son regard gli sser, les yeux grands ouverts, vers les masses d'ombre épaisse des meubles qui envahissaient la pièce «Q u'est-cequej'ai ? sedisait-il, le coeur lourd. Q ui sait? C'est l'angoisse crépusculaire», maisil s'étonnait denel'avoir encore jamais ressentie...

(U n Balcon en forêt, p. 120sq) 
On peut se demander si la scène décrite en (3) n'est pas racontée avec un pseudo-itératif, c'est-à-dire s'il ne s'agirait pas d'une scène qui ne s'est effectivement produite qu'une seule fois, et qui est malgré tout représentée avec cette visée itérative. Ici, rien de comparable à ce qui se produit chez Proust, et que $\mathrm{G}$ enette a analysé dans Figures III : nous sommes en présence d'un véritable itératif. Cette scène se produit quotidiennement, durant ces longues journées d'hiver où les hommes se trouvent dans une vacanceà la fois délicieuse et lourde. Ainsi, chaque jour, G range part pour d'enthousiasmantes promenades en luge avec $M$ ona, et à chaque fin de journée, avant de retourner à la «maison des hommes», G range cherche à apaiser ses angoisses (et celles de M ona), dans ces étreintes silencieuses...

C ette «angoisse crépusculaire» gagne progressivement Grange, sous l'influence de M ona (sensible «aux avertissements de l'heure et de la saison»). C ette crainte grandit peu à peu en $G$ range, elle ne lui est pas révélée d'emblée. C'est pourquoi le texteprécise que $G$ range «s'étonnait dene I'[l'angoisse crépusculaire] avoir encore jamais ressentie... » Les points desuspension et la négation sont l'indice de cette vérité progressive, au fil des jours, et au fur et à mesure que croît son attachement pour M ona.

En bref, cette scène condense maintes scènes semblables, et, surtout, présente ici une connaissance que $\mathrm{G}$ range a de ses sentiments excédant chacune de ces scènes prise isolément: en quoi la profondeur de perspective est quasi illimitée, puisque le savoir de $G$ range dépasse le cadre de cette scène ici racontée. Les procès rapportés par les imparfaits relèvent bien de la fréquence itérative, puisque les perceptions et, surtout, les pensées de plus en plus morbides qu'elles suggèrent renvoient à une accumulation de sai siesquotidiennes, la prescience de $G$ range dépassant en définitivela conscience qu'il a de chaque expériencequotidienne prise en elle-même.

Q uels enseignements tirer, sur la base de la confrontation de (2) et (3), relativement au rôle de l'itératif, et à son incidence sur la profondeur de la perspective du PDV ? D e fait, I'itératif est le moyen par lequel des évènements qui se sont répétés plusieurs fois sont exprimés sous la forme d'un condensé de multiples expériences, lequel synthétise alors beaucoup plus d'informations que ce ne serait le cas lors de chaque procès de perception, dans un cadrespatial et temporel déterminé. Si le focalisateur est identique, et si $S^{\circ}$ reste approximativement le même, en revanche, ces informations différentes qui résultent de visions différentes du même «objet», à des moments différents, se superposent, et sont pour ainsi dire «compactées», tout comme ces diverses temporalités setrouvent elles-mêmes «compactées» dansl'itératif. Le résultat en est une augmentation de la masse informationnelle du point de vue. Cette situation se produit lors de visions ${ }^{16}$ dans lesquelles, notamment, l'imparfait nejoue plus seulement un rôle de mise en relief, mais indique une vision itérative des perceptions ou des pensées focal isées. La charge cognitive de ces fragments s'en trouve sensiblement accrue. Cette nouvelle donne est-elle propre à l'expression de scènes focal isées, ou peut-elle être étendue à des pauses descriptives (portraits, ou descriptions de lieux eux-mêmes focali isés)?

\subsection{Portraits itératifs ou singulatifs}

N ousn'ignorons pas que le concept d'itérativité, en tant qu'il renvoie à l'analyse de la fréquence narrative, perd de sa pertinence dès qu'on sort de l'analyse des scènes. Par définition, la description, renvoyant le plus souvent à un état, paraît n'être concernée que par la fréquence itérative. La description est souvent saturée de traits itératifs, et il semble que cette itérativité soit un trait normal, voire indispensable, de toute description, au point qu'il soit impensable d'imaginer l'existence de descriptions avec une fréquence singulative:

D ansle récit classiqueet encorejusquechez Balzac, lessegments itératifs sont presque toujours en état de subordination fonctionnelle par rapport aux scènes singulatives, auxquelles ils donnent une sorte de cadre ou d'arrièreplan informatif [...] La fonction classiquedu récit itératif es donc assez proche de celle de la description, avec laquelle il entretient d'ailleurs des rapportstrès étroi ts: le «portrait moral» par exemple, qui est l'unedesvariétés du genre descriptif, procèdele plussouvent (voyez La Bruyère) par accumulation detraitsitératifs Comme la description, le récit itératif est, dansle roman traditionnel, au servicedu récit «proprement dit», qui est le récit ingulatif.

(Genette, 1972: 148)

Certes, $\mathrm{G}$ enette, à la fin de son analyse de la fréquence, prend soin de reconnaître que «la description est presque toujoursà la fois ponctuelle, durative et itérative, sans jamais s'interdire des amorces de mouvement diachronique» (G enette, 1972: 178), il n'en reste pas moins que, selon lui, la fréquence normée des descriptions est l'itérativité, et que la fréquence singulative est I'apanage quasi exclusif du «récit "proprement dit" » (ibid.: 148). Or la réalité est plus complexeque $G$ enette le laisse entendre, commele vérifiela comparaison de (4) avec (5):

(4) M aintenant qu'il [Grange] s'était un peu rapproché, ce n'était plus tout à fait une petite fille: quand elle [M ona] se 
mettait à courir, les hanches étai ent presque d'une femme; les mouvements du cou, extraordinairement juvéniles et vifs, étaient ceux d'un poulain échappé, mais il y passait par moments un fléchissement câlin qui parlait brusquement de tout autre chose, comme si la tête se souvenait toute seule de s'être déà blottie sur l'épaule d'un homme. Grange sedemandait, un peu piqué, si elle s'était vraiment aperçue qu'il marchait derrière elle: quelquefois el le s'arrêtait de côté sur le bord du chemin et partait d'un riredebien-être, commeon en adresseà un compagnon de cordée qui montederrière vouspar un matin clair, puis, des minutes entières, ellesemblait l'avoir oublié, reprenait son sautillement de jeune bohémienne et de dénicheuse de nids - et tout à coup elle paraissait extraordinairement seule, à son affaire, à la manièred'un chaton qui se détourne de vous pour un peloton de fil.

(U n Balcon en forêt, p. 54)

Cette première apparition de $M$ ona est présentée d'après la vision de $G$ range: ses perceptions et pensées, flottantes et intriguées, selimitent strictement aux informations visuelles dece dernier, ou, à tout lemoins, sont directement suscitées par ce que $G$ range observe en $S^{\circ}$ et $T^{\circ}$. II va de soi que des procès tels que «quelquefois elle s'arrêtait de côté sur lebord du chemin et partait d'un rire de bien-être» expriment la réitération, mais la saisie perceptuelle de ces derniers est comme sous la domination singulative du premier plan, les bornes temporelles de $\mathrm{T}^{\circ}$ étant étendues à la durée de cette première vision de $\mathrm{M}$ ona par $\mathrm{G}$ range. II ne faut donc pas confondre aspect itératif et fréquence itérative: il n'y a aucun douteà cesujet, cette description est bien celle dela première apparition de $M$ ona, de sorte quel'on se trouve ici face à un pseudo-itératif. En (4), l'imparfait traduit le regard distancié que porte le focalisateur sur cet être mystérieux, propre à engendrer un regard attentif et erratique. Rappelons à ce sujet ce qu'écrivait Pouillon de l'imparfait:

Par ce moyen on peut présenter l'action comme un spectacle. C'est là en effet le véritable sens romanesque de l'imparfait: ce n'et pas un sens temporel, mais, pour ainsi dire, spatial; il nous décale de ce que nous regardons. Cela ne veut pas dire que l'action est passée, car on veut au contraire nous y faire assister, mais qu'elle est devant nous, à distance et que c'est justement pour cela que nous pouvons y assister. Si les temps des verbes, comme la langue l'exprime, ont pour rôle premier d'exprimer desrapportstemporels, nous sommesen présenceici d'un cas où un rôle dérivé et plus subtil doit être rempli : exprimer un pur rapport deposition entrecequi est racontéet celui qui raconteou plutôt celui à qui c'est raconté; pour cela, on negarde, du décalagetemporel qu'exprimel'imparfait, que le sens général de décalage, sans le qualifier plus précisément.

(Pouillon [1946], 1993: 144sq)

Les renseignements que $G$ range nous donne sur M ona se cantonnent à ce que ce dernier observe lors de cette apparition, à travers une description d'actions au demeurant très imagée. Le caractère limité de la profondeur de perspective de (4) apparaît nettement, relativement à l'exemple suivant. En (5), en revanche, le portrait du capitaine Varin est un condensé de maintes visions de ce dernier, lors de ces dimanches ennuyeux que $G$ range passe auprès de son chef, en des temporalités différentes (T 1, T2, T 3...TN ). Le fait que Grange puisse se livrer à un portrait physique et surtout psychologique de son supérieur (alors que celui-ci reste sur un quant-à-soi obstiné, rétif aux épanchements, nefavorisant guère la connaissance de soi), le fait qu'il puisse affirmer qu'il ne «riait jamais», ne fréquentait pas Charleville, tout cela dénote un savoir du personnage-focalisateur excédant le savoir qu'il pourrait découvrir au «premier regard», de sorte qu'il est légitime de parler d'une profondeur de perspective quasi illimitée.

(5) Q uelquefois, après le déjeuner, à travers le sommeil des ruesdu dimancheouvrier, G range accompagnait un camarade jusqu'au train de Charleville, puisil passait au bureau de la compagnierégler quelquesdétails seservice. II y trouvait d'habitude le capitaine Varin fumant sa cigarette derrière des paperasses. Le visage était lourd, un peu carnassier sousla brosse dure et encore très noire, la narine flairante et épatée, la mâchoire large; au premier regard, on n'avait devant soi qu'un soudard à la tournure assez épaisse, mais c'était un soudard qui nebuvait pas, neplaisantait pas, neriait jamais, et, depuis que la division était montée en secteur, n'avait encore mis les piedsà la Place ducale de Charleville, où les officiersà tour de rôle allaient le dimanche traîner leurs guêtres. II menait sa compagnie avec une sécheresse glaciale et compétente, tenant aux hommeset aux officiersla bride courte, réglant lesaffaires en peu de mots, la voix brève, écoutant assez, ne discutant jamais. II est né pour lestylej'ordonne ou je metais- il a dû se tromper d'époque, ou se tromper d'armée, se disait Grange - que le capitaine intriguait - toujours étonné de ce bureau nu qui respirait durement la règle, rincé comme une porterie de couvent, où on nevoyait pas de sè èe pour levisiteur, et pas même une bouteille d'apéritif. Q uand il était seul à seul avec lui, G rangeparfois, quelquesinstants, sentait lecapitaineV arin plusproche, presque ouvert - non qu'il sedétendît: il travaillait toutela journée- non qu'il devînt plushumain: sa confidence 
était impersonnelleau point d'êtreglaçante; cequ'elecherchait était tout autrechoseque demettreà l'aise Lecapitaineparlait dela guerre. (U n Balcon en forêt, p. 45)

Ainsi, les portraits focal isés peuvent s'accommoder d'une fréquence tantôt singulative, commeen (4), tantôt itérative, commeen (5). II est habituel des'appuyer sur ces perceptions réitérées pour conclure à un PDV du narrateur, au mépris de la référenciation. S. Vogel eer a tendance à considérer que de tels imparfaits, si habituels, excluent le «vrai » point de vue perceptuel (correspondance privée). Cette conception repose sur l'idée d'une saisie perceptuelle hic et nunc (ce qui n'est pas niable), saisie qui semble devoir être unique, non réitérable (ce qui est plus discutable).

$M$ ais en vertu de quels principes une saisie perceptuelle du focalisateur condensant de multiples saisies par lemême focalisateur se changerait-elle en un PDV du narrateur? En effet, en (3) ou en (5), c'est certes toujours le mêmenarrateur qui raconte (voix narrative), mais son récit appréhende les évènements d'après le PDV de G range (mode narratif), et I'usage de la fréquence itérative n'enlève rien à ce fait, en sorte que la fréquence relève bien de la voix narrative, et aussi du mode, puisque cette réitération des procès est vécue, mais aussi et surtout reconnue comme telle par le personnagefocalisateur lui-même C'est cequeFontanillefaisait justement observer à propos del'exemple des tromperies successives de $M$ anon, racontées par D es G rieux: Fontanilleremarquequ'il s'agit là d'une itération narrative, mais pas d'une itération discursive, ce qui explique que si le lecteur est capable de reconstituer l'itération sur la base des instructions fournies par le narrateur, en revanche, D es G rieux n'y voit pas la répétition à l'identique de ses malheurs ${ }^{17}$. Ce n'est pas le cas, en (5), et c'est pourquoi il y a ici uneitération discursive, qui est indissociabledu PDV de Grange, et qui est àl'origine de l'accroissement de la profondeur de perspective de ce dernier.

Ainsi, la fréquenceitérativen'est pasl'apanagedu narrateur; dès lors qu'elle concerne le PDV du personnage, elle a pour conséquence pratique de densifier la profondeur de perspective de son PDV. C ette conclusion se trouve confirmée par l'examen d'une nouvelle série de pauses descriptives, consacrées, cette fois-ci, à des topographies.

\subsection{Descriptions de lieu itératives ou singulatives}

Les topographies focalisées suivantes confirment le rôle de la fréquence dans la profondeur de perspective du point de vue du personnage: en (6), la fréquence singulative va de pair avec la profondeur de perspective limitée de la vision du lieu, alors que la fréquence itérative, en (7), va de pair avec la profondeur de perspectivequasi illimitée de la vision du lieu.

(6) - Vous êtes chez vous, fit le capitaine Vignaud.

La maison fortedesH autesFalizesétait un desblockhausqu'on avait construitsen pleineforêt pour interdireaux blindésl'accès des pénétrantes descendant de l'A rdenne belge vers la ligne de la M euse. C'était un bloc de béton assez bas, où l'on accédait versl'arrière par uneporteblindéeet un sentier en chicanequi traversait une petite plantation de barbelés serrée contre le blockhaus à la manière d'un carré de choux. On l'avait barbouillé à la diable d'un vert olive délavé qui sentait la moisissure: des espèces de dartres fongueuses entretenues par la touffeur du sousboislaissaient suppurer sur lesparois destaches humides, commesi on y avait étendu tous les jours des draps mouillés. L'avant du blockhausétait trouédedeux embrasures; I'une, étroite, pour unemitrailleuse, l'autre, un peu pluslarge, pour un canon anti-char. Sur ce bloc trapu reposait comme sur un socletrop étroit l'étage débordant d'une mai sonnetteoù on accédait latéralement par un escalier de fer ajouré, pareil au fire-escape des maisons américaines: c'était le logement de la garnison minuscule. (U n Balcon en forêt, p. 20)

En (6), la description de la maison forte est d'une profondeur de perspective limitée, puisque strictement cantonnée à $\mathrm{S}^{\circ}$ et $\mathrm{T}^{\circ}$. $\mathrm{C}^{\prime}$ 'est la premièrefois que $\mathrm{G}$ range découvre son domaine, et ce que le lecteur en connaît suit le mouvement de la découverte du blockhaus, au fur et à mesure que $G$ range s'en approche et tourne autour. En effet, I'indéfini «on», sujet des verbes de perception, se rapporte par inférenceà $\mathrm{G}$ range, qui est le personnagesaillant en contexte: c'est à lui ques'adressele capitaine Vignaud, dont les propos sont uneinvitation au nouveau «maître des lieux» à découvrir son domaine. O n peut éventuellement considérer quel'indéfini renvoie à tous les spectateurs potentiels qui découvrent le blockhaus, c'est-à-dire l'ensemble des subordonnés de Grange, en sorte que l'instance focal isatrice est singulière, ou plurielle. D ans les deux hypothèses, la vision (du ou des personnages) est limitée: c'est celle de militaires découvrant un ouvrage militaire, et portant un regard de militaires sur ce qu'ils découvrent; c'est celle d'hommes qui nesesont pas encore appropriés la maison forte. Aussi cette découverte progressive se borneà des informations seulement accessibles en $\mathrm{S}^{\circ}$ et $\mathrm{T}^{\circ}$, $\mathrm{C}^{\prime}$ est-à-direau moment de la première découverte de l'endroit. II n'en va pas de même en (7).

(7) Lorsqu'il était rentré dans sa chambre, Grange lisait un moment à la lueur d'une mauvaise lampe voleuse qu'il 
accrochait par une griffe au-dessus de sa table à la cloison de bois, mais les libations d'après dîner l'énervaient, et, surtout lorsque le temps était sec et qu'il y avait de la lune, il sortait pour une courte promenadeavant dese coucher. La nuit de la forêt n'était jamaistout à fait noire. D u côté de la M euse, très loin, le rebord opposé de la vallée dans les trouées des arbres blanchissait vaguement par moments d'une espèce de fausse aurore, une palpitation soyeused'éclairsmouset gras, pareilsà ces grosses bulles de lumière qui crèvent par intervalles audessus des vallées de hautsfourneaux: c'était le béton des casemates, pour lequel on mettait maintenant les bouchées doubles, qu'on coulait la nuit à la lueur dessunlights. D u côté de la frontière, où le plateau peu à peu s'élevait, on voyait perler un à un et gli sser quelquesinstants dansla nuit de petits points de lumièrequi sépanouissai ent sans bruit et balayaient la crête des taillis d'un rayon rapide: les automobiles belges, qui roulaient dans la paix d'un autre monde au travers des clairièresplusaéréesoù l'Ardennepeu à peu semorcelait. Entre ces deux franges que la nuit soudain alertait vaguement, le T oit (c'était le nom que donnait G rangeà ce plateau de forêts suspendu au-dessusdela vallée) restait plongédansuneobscurité profonde. La laie sallongeait à perte de vue comme une route fantôme, à demi phosphorescente entre les taillis sous son poudrage de gravier blanc. (U n Balcon en forêt, p. 38)

(7) présente une vision de profondeur quasi illimitée, dans la mesure où le spectacl e que rapporte $G$ range dépasse cequ'il pourrait percevoir à chacune de ses promenades. Son savoir ne provient d'ailleurs pas seulement de ses observations successives: en effet, Grange ne peut pas voir depuis les Falizes, ou depuis un point quelconque du Toit, le «béton des casemates» qu'on est en train de couler. II s'agit donc d'informations glanées auprès de ses supérieurs, par conséquent d'informations qui excèdent le cadre spatial et le cadre temporel de cette vision.

Ces quelques exemples de portraits, descriptions de lieux et de scènes témoignent des corrélations entre la profondeur de perspective et la val eur itérative des visions. Le point de vue du personnage corrélé à l'itératif s'accompagne d'une augmentation tendancielle du volume du savoir des visions (et, assez souvent, va de pair avec une augmentation tendancielle de leur longueur). En l'occurrence, le point de vue du personnagen'est pas nécessairement limité, comme on se plait à le dire trop souvent ${ }^{18}$. Certes, la restriction de champ est la perspective la plus fréquemment adoptée par le personnage, mais ce n'est pas nécessairement la seule. En cette matière, il convient de distinguer l'approche ontologique de l'approche linguistique: certes, ontologiquement, voire phénoménologiquement, la perspective des individus est limitée; mais linguisiquement, rien n'interdit à un sujet quelconqued'adopter une perspective qui ne soit pas bornée par une saisie hic et nunc, et donc de compacter des temporalités différentes ${ }^{19}$.

Ainsi, entre autres facteurs susceptibles d'indiquer une vision étendue, la fréquence itérative joue un rôle notable (et notablement sous-estimé). $0 \mathrm{n}$ peut toutefoissedemander si cette dimension ne concerne que le point de vue du personnage, ou si elle se concrétise également avec le point de vue du narrateur, et, si oui, quels en sont les effets.

\section{Fréquence itérative et point de vue du narrateur}

Un mot, au préalable, sur ce concept. Le lecteur attentif n'aura pas manqué de relever que nous avons parlé de point de vuedu narrateur. Peut-être aura-t-il ététentéde conclure que c'était une autre manière de parler de la focalisation zéro de $G$ enette. En réalité, ce changement de dénomination renvoie à un changement de conception: $G$ enette définit la focal isation zéro tantôt comme une absence de focal isation, tantôt comme une focalisation variable. Pour nous, le point devuedu narrateur existe bel et bien, dès lors queles perceptions ou les pensées représentées ne coréfèrent pas à un personnage saillant. Les perceptions ou les pensées représentées dans les seconds plans tran sforment al ors le narrateur en un énonciateur, y compris le narrateur anonyme, comme le montrent nos exemples suivants: en effet, ce narrateur anonyme, même dénué d'identité et de rôle actanciel ou actoriel, exprime des valeurs qui découlent de ses choix dans la référenciation (dans l'évaluation ou la modalisation). En ce sens, le narrateur-énonciateur responsable des perceptions et des pen sées représentées dans les seconds plans est susceptible de jugements plus ou moins subjectivants, al ors que le narrateur-locuteur des premiers plans est objectivant, puisque les faits sont rapportés comme indépendamment de lui 20.

L'examen du rôle de l'itératif dans la profondeur de perspective du narrateur confirme que, si omniscience il y a, ellenetient pasà sa nature ontologique, commeV itoux s'était justement attaché à le dire (1982). La preuve en est fournie, a contrario, par le fait que, dès lors que le point de vue du narrateur s'accompagne de la fréquence singulative, la perspective qui est construite est limitée par $\mathrm{S}^{\circ}$ et $\mathrm{T}^{\circ}$ et, de ce fait, cette limitation de la profondeur de perspective rend insanel'idée de l'omniscience systématiquedu narrateur. Ce résultat prend non seulement à contre front la théorie traditionnelledel'omniscience... mais surtout, il confirmebien le rôle del'itératif dans la construction d'une profondeur de 
perspective quasi illimitée (pour le personnage) et illimitée (pour le narrateur) ${ }^{21}$, en manifestant que cette profondeur de perspective tient à des critères textuels. L'omniscience, qu'il n'est pas question de rejeter, mais qu'il convient de mieux fonder, résulte moins de l'encyclopédisme du narrateurfocalisateur (il existe aussi des personnages-focal isateurs très savants, alors que certains narrateursfont preuve de beaucoup d'ignorance) que du fait de savoir si les perceptions (et le savoir qu'elles véhiculent) sont limitées, ou non, à $S^{\circ}$ et $T^{\circ}$ (et donc, selon une logique naturelle semblable au post hoc, ergo propter hoc à l'œuvre dans le récit), limitées, ou non, par $S^{\circ}$ et $T^{\circ}$. Pour éviter d'inutiles redites, nous nous contenterons ici d'exemplifier rapidement le lien entre fréquence et profondeur de perspective narratoriale.

\subsection{Fréquence itérative \\ et profondeur de perspective illimitée}

Ainsi, (8) et (9) illustrent-ils le lien entre la fréquence itérative et la perspective illimitée de pauses narratives, alors que la profondeur de perspective limitée de (10) est en rapport avec le caractère singulatif de la narration.

(8) C'était en effet la light-house des C asquets. Un phare au dix-neuvièmesiècleest un haut cylindreconoïde demaçonnerie surmontéd'unemachineà l'éclairagetout scientifique. Lephare des Casquets en particulier est aujourd'hui une triple tour blanche portant trois châteaux de lumière. Cestrois maisonsà feu évoluent et pivotent sur des rouages d'horlogerie avec une telle précision que I'homme de quart qui les observe du large fait invariablement dix pas sur le pont du navire pendant l'irradiation, et vingt-cinq pendant l'éclipse[...]

Au dix-septième siècle un phare était une sorte de panache de la terre au bord de la mer. L'architecture d'une tour de phare était magnifique. $0 \mathrm{n}$ y prodiguait les balcons, les balustres, les tourelles, les logettes, les gloriettes, les girouettes. Ce n'étaient que mascarons, statues, rinceaux, volutes, rondes-bosses, figures et figurines, cartouches avec inscription.[...] M aisle phare des Casquets n'était point de cette mode.

C'était à l'époque un simple vieux phare barbare, tel que H enri I l'avait fait construireaprèsla perdition dela Blanche $\mathrm{N}$ ef, un bûcher flambant sous un treillis de fer au haut d'un rocher, unebraisederrièreunegrille, et unecheveluredeflamme dansle vent.

Le seul perfectionnement qu'avait eu ce phare depuis le douzièmesiècle, c'était un soufflet deforgemisen mouvement par une crémaillèreà poids de pierre, qu'on avait ajustéà la cage à feu en 1610.

(V. H ugo, L'H omme qui rit, L'Intégrale III, Paris, Seuil, 1963, p. 227)
Cette vision du phare est en effet illimitée puisque, alors que l'action se passe en $1690\left(=T^{\circ}\right)$, le narrateur évoque les phares d' «aujourd'hui », c'est-à-direau dix-neuvième siècle, puisse situe antérieurement à $T^{\circ}$ en évoquant l'amélioration technique de 1610. Pareillement, alors que $S^{\circ}$ correspond à la vue du phare des C asquets depuis l'ourque en détresse, le narrateur évoque la vue du phare depuis n'importe quel bateau d'aujourd'hui, sans compter la description du phare d'E ddystone, que nous n'avons pas rapportée. C es faits suffisent à indiquer une vision illimitée. C ertes, les connai ssances encyclopédiques du narrateur se surajoutent, mais elles ne sont pas|'élément décisif du caractèreillimitéde cettevision, tout comme en (9):

(9) Dans son impassibilité, peut-être seulement apparente, éaient empreintes les deux pétrifications, la pétrification du coaur, propreau bourreau, et la pétrification del'esprit, propre au mandarin. $0 \mathrm{n}$ pouvait affirmer, car le monstrueux a $\mathrm{sa}$ manière d'être complet, que tout lui était possible, même sémouvoir. Tout savant est un peu cadavre; cet homme était un savant. Rien qu'à levoir, on devinait cettescience empreinte dans les gestes de sa personneet dans les plis de sa robe. C'était unefacefossiledont lesérieux était contrariépar cettemobilité ridéedu polygl ottequi va jusqu'à la grimace. Du reste, sévère. Rien d'hypocrite, mais rien de cynique. U n songeur tragique. C'était I'homme que le crimea laissé pensif. II avait le sourcil d'un trabucaire modifié par le regard d'un archevêque.

(L'H omme qui rit, p. 216)

D ans ce portrait moral, lenarrateur hugolien jouece rôle prédictif si particulier. Le narrateur, dans des phrases déclaratives affirmatives, révèle sans coup férir la nature profonde du personnage, sur la base de son seul physique, et de son comportement. Les informations fournies en $\mathrm{S}^{\circ}$ et $\mathrm{T}^{\circ}$ sont insuffisantes pour justifier, premièrement, ces interprétations, deuxièmement, leur caractère péremptoire: «tout lui était possible, même s'émouvoir », vaut autant pour le passé du personnage que pour son avenir; le caractère certain de ce qui a àla foisunevaleur analeptique et une val eur proleptique est, ainsi que Lintvelt l'a remarqué, une des marques les plus solides de la profondeur de perspective illimitée. D e plus, il est vrai que ces commentaires, même s'ils ne sont pas au présent gnomique, visent à une général itédont rend notamment comptel'usage du défini dansl'énoncé «c'était l'homme que le crime a laissé pensif»: cette généralisation, en congruence avec un abondant vocabulaireabstrait, s'accompagne bien de cette vision illimitée, combinée avec la dimension itérative. 


\subsection{Fréquence singulative}

\section{et profondeur de perspective limitée}

Les exemples (8) et (9) offrent un contraste saisissant avec (10), marqué par la dimension singulative de la scène:

(10) La crique, murée de tous les côtés par des escarpements plus hauts quellen'était large, éait de minute en minute plus envahie par le soir ; la brumetrouble, propreau crépuscule, s'y épaississait; c'était comme une crue d'obscurité au fond d'un puits; la sortie de la crique sur la mer, couloir étroit, dessinait danscet intérieur presquenocturneoù leflot remuait unefissure blanchâtre. II fallait être tout près pour apercevoir l'ourque amarrée aux rocherset commecachéedansleur grand manteau d'ombre. U neplanche jetée au bord à une saillie basse et plate de la falaise, unique point d'où l'on pût prendre pied, mettait la barque en communication avec la terre, des formes noires marchaient et se croisaient sur ce pont branlant, et dans ces ténèbres des gens s'embarquaient.

(L'H ommequi rit, p. 203)

Le narrateur-focalisateur (c'est le même, dans tous nos exemples) adopte ici une vision limitée à ce que verrait un observateur anonyme en $\mathrm{S}^{\circ}$ et en $\mathrm{T}^{\circ}$ : il n'y a ni analepse ni prolepse, et le focalisateur évoque «ces» ténèbres, «ce» pont branlant qu'il voit de «tout près». Les démonstratifs ont une val eur ostensive-situationnelle limitée par $S^{\circ}$ : $c^{\prime}$ est pourquoi le mode de donation des référents, à la fin de cet extrait, évoque «desformesnoires», «desgens», sans plus deprécision.

Cette profondeur de perspective «à géométrie variable» permet de rendre compte des variations de perspectivejouant à l'intérieur d'un même extrait, sans qu'il soit besoin d'alléguer un changement de focalisation, comme c'est trop souvent le cas lorsque l'on invoque le passage d'une focal isation externe à une focal isation zéro 22 :

(11) Q uel quefût l'aspect du temps, lesêtresqu'allait emmener I'ourquebiscayennen'en pressaient pas moins le départ. IIsfaisaient au bord de la mer une sorte de groupe affairé et confus, aux allures rapides. Lesdistinguer l'un del'autreétait difficile. Impossibledevoir s'ilsétaient vieux ou jeunes Lesoir indisinct les mêlait et les estompait. L'ombre, cemasque, éait sur leur visage C'étaient des silhouettes dansla nuit. I Isétaient huit, il y avait probablement parmi eux uneou deux femmesmalaiséesà reconnaître sous les déchi rures et les loques dont tout le groupe était affublé, accoutrementsqui n'étai ent ni desvêtements defemmes, ni des vêtements d'hommes. Les haillonsn'ont pas de sexe. U ne ombre plus petite, allant et venant parmi les grandes, indiquait un nain, ou un enfant.

C'était un enfant.

(L'H ommequi rit, p. 203sq)
(11) illustre en effet ce passage, d'une phrase à l'autre, d'une vision externelimitée à une vision externe illimitée: le narrateur prend soin de nous dire qu'il était «impossible de voir » l'âge des personnages, que «l'ombre, ce masque, était sur leur visage», qu'il faisait nuit. Cette situation est encore réaffirméeà la fin del'extrait, puisquel'avant-dernièrephrase évoque une ombre, sans préciser davantage s'il s'agit d'un enfant ou d'un nain, les conditions météorologiques ne permettant pas d'en dire davantage, en $\mathrm{S}^{\circ}$ et $\mathrm{T}^{\circ}$, dans le code réaliste dominant auquel se plie le narrateur. $0 \mathrm{r}$, dans la phrase suivante, sans aucune transition, dans un nouvel alinéa, le même narrateur à l'origine de ces deux visions valide I'hypothèse de l'enfant. C ette dernière vision, exprimée par le biais d'un présentatif et d'un imparfait de mise en relief, à valeur expériencielle mémorielle, contribue à créer un effet dedramatisation, afin defaire ressortir par contrastel'enfant sur les autres personnages. Cette mise en relief, exhibée par la concision de la phrase, en clôture du premier chapitre du premier livre, fait entendre, par la matérialité de son dire, combien l'enfant sera au centre du récit à venir. Incontestablement, ledire- danslequel l'organisation typographique et la segmentation du texte jouent un rôle important - est créateur de sens, et construit les interprétations présentes et à venir en créant un fort horizon d'attente. Ces intentions communicationnelles expliquent la suspension momentanée du pacte de croyance réal iste, et lechangement de profondeur de vision est justifiénon par un changement de référents, ou de focalisateur, mais par des choix scripturaires 23 .

\section{L'influence de l'itératif dans l'expression de la profondeur des perspectives narratives du personnage et du narrateur}

1) N otre première remarque concerne le rôle notable de la fréquence itérative dans l'expression linguistique de la profondeur de perspective: en effet, les visions itératives du personnage-focalisateur ou du narrateur-focalisateur entraînent une profondeur de perspective quasi illimitée ou illimitée. A contrario, les visions singulatives témoignent d'une profondeur de perspective limitée, tant pour le personnage focalisateur que pour lenarrateur-focalisateur. C ette influence de la fréquence sur la profondeur de perspective a l'insignemérite de mettre en lumière le fait quela restriction de champ et l'omniscience ne sont pas des données intangibles et obligées, mais, au contraire, des données variables, tributaires dechoix narratologiques et, en dernière instance, de modalités linguistiques.

C'est d'ailleurs pour témoigner au mieux de ce que les changements de visions et de profondeur sont tributaires 
des stratégies de l'écrivain, que nous avons cantonné nos exemples à une seule œuvre, L'H omme qui rit (comme nous I'avons fait précédemment, pour les mêmes raisons, avec U n Balcon en forêt). L'appartenance de nos exemples à une seule et même œuvre montre combien le même narrateur, voire le même focalisateur (dans le cas d'U n Bal con en forêt), dans la même œuvre, est capable de varier les visions et les profondeurs de perspective, «tout naturellement», en fonction des référents, des situations, des intentions communicationnelles, etc., sans qu'il soit nécessaire de parler hors de propos d'altérations du principe del'omniscience du narrateur, ou d'invoquer un brusque passage en focalisation externe...

2) N otre deuxième remarque propose de préciser deux paramètres importants (et trop souvent confondus) rendant compte de la nature complexe de la profondeur de perspective. Celle-ci comporte une masse de données importantes, surdéterminées, relativement à $S^{\circ}$ et à $T^{\circ}$. $L^{\prime}$ 'on peut en effet donner des informations (peu ou beaucoup) tout en se «limitant» àcequ'il est matériellement possibleà un observateur lambda de voir (et d'inférer) dans un espace et une temporal ité donnés, ou l'on peut au contraire donner des informations (peu ou beaucoup) qui débordent de ce cadre spatiotemporel. Pour rendre compte de la première situation narratologique, on mettra en avant le critère de l'étendue, de plus ou moins grande amplitude sel on la quantité d'informations fournie, cettequantitévariable d'informations étant toujours limitée par le cadre spatio-temporel. Pour rendre compte dela seconde situation, on mettra en avant le critère de la profondeur. Ce critère qualitatif de la profondeur du savoir, illimitée ou quasi illimitée, apparaît dès lors que les informations excèdent la perception ou le savoir d'un observateur anonymedans un cadre spatio-temporel donné. Ceci revient à direquel'extension de la profondeur de perspective est notamment corrélée à la présence de l'itératif, puisque cette fréquence se caractérise par le fait que le récit condense des temporalités différentes, et donc compacte les savoirs accumulés au cours des diverses saisies perceptuelles, ou permet l'émergence de savoirs qualitativement nouveaux sur la base de la conscience de cette réitération discursive.

3) N otre troisième remarque a trait à une sorte de corré lation entrel'itératif et la longueur des visions: cephénomène s'expliquesans doute par lefait quel'itératif permet derendre compte de plusieurs procès dans leur continuité, et dans le même temps d'en rendre compte en les analysant en autant d'unités discrètes quele souhaitele producteur del'énoncé24. T outefois, lefait que, dans nos exemples, les visionsitératives soient développées sur de plus longues portions textuelles queles visions singulatives ne doit pas pour autant amener à conclure qu'une scène singulative doit nécessairement être de faible longueur, ou qu'elle doit nécessairement renvoyer à une profondeur de vision limitée. T out cela dépend de multiples paramètres qui se laissent mal résumer en uneformule de «grammaire du récit»; ainsi, il est prévisible que la nature du personnage focal isateur, la nouveautédes «objets» observés, les intentions communicationnelles de l'écrivain, les lois d'un genre, etc., peuvent entrer en jeu.

4) Ces remarques précédentes sur la variabilité des profondeurs de perspective nous conduisent à une dernière conclusion concernant la nature de quel ques-unes des contraintes pesant sur le choix de la profondeur de perspective. Peut-on continuer à affirmer que la profondeur de perspective illimitée est la caractéristique dominante des visions du narrateur? O ui, s' l'on a conscience que «essentielle» ne signifie pas «unique». II est d'abord crucial deréaffirmer, contrelemythe tenace de «|'omniscience du narrateur», quetoutes les visions et quetousles niveaux de profondeur sont accessiblesau narrateur (et, mutatismutandis, la réponse vaut pour la restriction de champ qui n'est pas l'unique profondeur de perspective du personnage-focali sateur, mêmesi c'est la plus fréquente).

Si toutefois la profondeur de perspective illimitée du narrateur-focalisateur parait dominer sur les visions limitées, on peut expliquer cefait par l'hypothèse dela «commodité», surtout valable en régime de fiction réaliste. Le parti pris d'un point de vue du personnage ou celui d'une profondeur de perspective limitée pour le point de vue du narrateur impliquent dans les deux cas une sélection des informations, et donc des contraintes, qui peuvent peser lourdement sur la conduite du récit, dans la mesure où le souci de vraisemblabiliser les informations fournies par lebiais d'une restriction dechamp peut amener à des complications del'intriguedont la motivation technique est la «naturalisation» des informations transmises par ce canal. C'est notamment pour éviter ces complications factices quel'écrivain recourt plus ou moins souvent à des visions illimitées du narrateur.

Cetteprofondeur de perspective est donc à la fois variable et contrainte, dans le régime de fiction réaliste: il est possible de passer d'une profondeur de perspective limitée à une perspective illimitée (c'est le mouvement «naturel » de l'inconnu au connu), mais l'inverse nel'est pas (on ne peut pas dire à la fois $A$ et non-A). T outefois, cette convention joue dans les limites suivantes:

a) Le passage d'une perspectiveillimitée à une perspective limitée n'est pas possible si la vision est celle du même référent, par le même focalisateur sauf si I'on change de focali- 
sateur, ou si I'on change de destinataire: dèslors le problème nese poseévidemment plus ${ }^{25}$. En revanche, si les visions du même focalisateur portent sur des référents différents, il est al ors possible de passer d'une vision illimitée d'un référent à unevision limitée d'un autre référent, sansque soient notamment violées les loisd'informativitéet d'exhaustivitéde G rice, pour autant que ces lois soient transposables sans modification au régime de la fiction réaliste.

Si le narrateur choisit cependant de s'en tenir le plus possible à une seule profondeur de vision, cela dépend de choix scripturaires, maisnon de règles narratologiques. C'est évidemment ce qui se produit avec ce que l'on appelle couramment et abusivement la focalisation externe: lenarrateur anonyme s'en tient (autant que possible, tant il s'agit d'un état instable) à des visions les plus limitées possible, tout en faisant le choix complémentaire d'une expression la plus objectivante possible.

b) II n'en demeure pas moins que le choix d'un point de vue, d'une vision, d'une profondeur de perspective, dès lors qu'il opère pendant une certaine durée, produit son propre horizon d'attente: il existedonc des «contraintes» internesà I'œuvre, relativement indépendantes des choix scripturaires del'écrivain, et avec lesquelles ce dernier doit compter. $M$ ais il est prématuré d'avancer des propositions solides sur ces niveaux, bien difficiles à déterminer dans l'absolu: parfois, plusieurs paragraphes de suitesuffisent pour créer cet horizon d'attente; dans d'autres cas un chapitre ou une partie de chapitre sont nécessaires.

En définitive, ces contraintes-là existent bel et bien, mais elles ne semblent cependant pas de nature à empêcher l'écrivain de choisir telle profondeur ou de passer d'une profondeur à une autre. Et, on le devine, ces contraintes pèsent encore plus fortement pour le point de vue du personnage, marqué par des conceptions réalistes de toutes sortes. $M$ ais, si fortes que soient ces contraintes psychologiques ou idéologiques, quels que soient «le parti pris des choses» et «le comptetenu des mots», I'écrivain se posetoujours la question du sens et de sa construction textuelle, dans une dialectique incessante entre contraintes et liberté.

$D$ ans cette dialectiquelà, les liaisons et déliaisons entre la fréquence itérative et la profondeur de perspective des différents points de vue jouent un rôle appréciable (hélas trop sous-estimé!) sur le plan narratologique comme sur le plan interprétatif.

\section{N OTES}

1. Cequi n'est pasla mêmechose, danslamesureoù toutedescription n'est pasforcément focal isée, et où tout point devueneselimitepasnécessairement à des pauses descriptives.

2. N ous voulonsici approfondir une réflexion amorcée dans les pages 160 165 de notre Construction textuelle du point de vue (1998).

3. D euxinstances, et non trois: il n'y a pasd'instanceénonciativeautonome (autrequelenarrateur et lepersonnage) susceptibledefonder unesoi-disant focalisation externe: cf. Rabatel 1997a, et 1997b, chap.12. Tout au plus peut-on considérer, par commodité de langage, que les focalisateurs (personnage ou narrateur) adoptent à certainsmoments des visions externes des objets de discours. $M$ ais, comme on l'a montrédans le premier chapitre de La Construction textuelle du point de vue, il s'agit vraiment d'une commoditédelangageà valeur métaphorique, tantl'expression linguistique des perceptions montre que ce qui est «interne» et «externe» est inextricablement mêlé. V oir également «Q uand voir c'est (faire) penser», 1999, à paraitre dans les Actes du colloque international de Lyon sur les relations intersémiotiques.

4. Pour uneapprocheglobaledela perspectivenarrative, cf. Lintvelt, 1981. 5. LePDV est un parasynonymedefocalisation narrative. La dénomination de PDV a l'avantage d'être utilisée hors du domaine français, et ne fait pas doublon avec celle de focalisation linguistique (mise en focus). En outre, ce changement denom marque (au-delà dela dette) une rupture avec $G$ enette, qui setraduit notamment par l'abandon delafocalisation externe, et par une définition nouvelle de la focalisation zéro.

6. En (1), lesénoncés en italiques correspondent au PD V. N os exemplesse limitent àl'analysedu PDV dansles récitshétérodiégétiques; celan'implique pas que nos conclusions ne soient pas transposables pour les textes homodiégétiques, l'opposition locuteur-énonciateur étant égal ement pertinente pour ces récits. Pour uneétudedu PDV dansles récitshomodiégétiques, voir Rabatel (2000).

7. Cf. Combettes (1992) pour la définition des premiers et seconds plans en français.

8. Cf. infra, notre conclusion, pour une définition plus précise de la profondeur de perspective. En (1b), les fragments en caractères romains gras renvoient à des informations qui dépassent le savoir d'un observateur lambda, qui découvrirait la scène.

9. Cf. len ${ }^{0} 102$ de L anguefrançaise, dirigépar P. D endaleet L. T asmowski, $1994: 4-5$. Les principal es sourcesdu savoir sont laperception, lesinférences ou l'emprunt à autrui (par exemple par le biais d'une discussion).

10. Genette, 1972: 148.

11. L'aspect itératif est marquépar lesémantismedecertainsverbes(ressasser), de certains préfixes (redire) ou affixes (criailler), ou, le plus souvent, par des compléments circonstanciels de temps.

12. N ous analyserons plus en détail cette déliaison avec l'exemple (4).

13. $\mathrm{S}^{\circ}$ et $\mathrm{T}^{\circ}$ se définissent par rapport à l'énoncé, et non par rapport à l'énonciation. Ce cadre spatio-temporel, qui sert de cadre de référence au point de vue, est fourni par et dans le premier plan du texte.

14. Au sens où C. Kerbrat-O recchioni parle de «récepteur additionnel», dans Les Interactions verbales.

15. Leconcept desaisieperceptuellesubjectiverenvoieàl'origineénonciative du procèsperceptuel, distinctedecelledu narrateur. Cetteorigineénonciative n'implique pas automatiquement que l'expression du PDV soit surchargée de subjectivèmes, mêmesi c'est souvent le cas. Sur la nécessité de distinguer origineénonciativeet expression subjectivante(ou objectivante) du focalisé, nous renvoyons à Rabatel, 1997b, chap.12, et, plus particulièrement, à Rabatel, 1998, chap. 2, 3e partie.

16. Le concept de vision renvoie à l'étude du «focalisé» (M. Bal, 1977), autrement dit decequi est perçu ou «repéré» (Guillemin-Flescher, 1984 :74) par le sujet-focalisateur. Le concept de point de vue est réservé plutôt au repéragedel'énonciateur auquel coréfèrent lesvisions. Cetteopposition n'a pas d'intérêt autre que didactique, car, sur le plan théorique, il est illusoire 
d'opposer radicalement ancrage énonciatif, d'un côté, et référenciation des objetsfocalisés, del'autre, puisquec'està partir descaractéristiquesspécifiques de la référenciation des objets que l'on peut «remonter » au repérage de la source énonciative. Autrement dit, vision et point de vue appréhendent le mêmephénomèneà partir deson aval (vision) ou desa source(point devue) : l'essentiel est de ne jamais... focaliser sur l'un au détriment de l'autre...

17. Cf. Fontanille, 1989: 42-43, et Rabatel, 1997b: 75-85.

18. Cf. Bal, 1977: 42 ; Lintvelt, $1981: 44$;V itoux, 1982 : 360-364; G enette, 1983.

19. Cette possibilitélinguistique d'exprimer une profondeur de perspective variable est également rendue possible par le fait que le personnagefocalisateur peut accéder aux pensées des autres personnages, dans des monologues intérieurs ou dans des récits enchâssés, et que, sur le plan de l'expression, aucun des mécanismes linguistiques del'investigation psychologique ne distingue le personnage du narrateur (qu'il s'agisse du psychorécit, du monologue intérieur, du discours indirect libre, de l'emploi des verbes du discours attributif, etc.) comme nous l'avons montré dans $U$ ne Histoire du point de vue, 1997b : 260-264.

20. Certes, il existedes fragmentsnon focalisés (par exempledes perceptions qui figurent danslepremier plan du texte, ou desperceptionsqui nesont pas représentées), maiscela n'autorise pas à conclureàl'absence de point de vue du narrateur, puisqu'il existe, à côté de ces fragments, des perceptions ou pensées focali sées par le narrateur: sur ce point, cf. Rabatel, 1998.

21. La distinction que nous établissons entre profondeur de perspective quasi illimitée et illimitée tient au fait qu'il y a bien une différence de visée pragmatique, deforceillocutoire, deconfiancedu lecteur, bref, unedifférence d'autoritémodulable sel on quelesinformations (et les évaluations, modalisations qui les accompagnent) émanent des personnages, ou du narrateur. 22. Cf. Rabatel, 1997a: 92-95.

23. On pourrait également considérer quel'évocation des femmes est aussi un indiced'unevision illimitéedu narrateur, danslamesureoù leur présence ne se laisse en rien distinguer par des haillons «qui n'ont pas de sexe». M ais il faut souligner quecetteprésenceest évoquéesouslaformed'unehypothèse ( «probablement» parce que les formes permettent cette inférence en $\mathrm{S}^{\circ}$ et en $\left.\mathrm{T}^{\circ}\right)$, alors que celle de l'enfant est une affirmation sur laquelle ne plane aucun doute: nous ne sommes pas sûrs qu'il y ait des femmes (la suite confirmera la présence d'une Basquaise et d'une Irlandaise), mais nous sommes certains qu'il y a un enfant, et non un nain.

24. D 'un point devuesémantique, commel'écrit M aingueneau, «l'itération associe deux traits qui seraient contradictoires à un même niveau: la continuité et la discontinuité. $D$ 'un côté, en effet, l'itération délimite un ensemble de procès saisi globalement, de l'autre elle suppose l'analyse de cette totalité en unités discrètes» (M aingueneau, 1986: 64).

25. L'impossibilité de passer d'une profondeur illimitée à une profondeur limitée du même référent peut néanmoins se trouver réalisée, dans des parodiesdel'écritureréaliste. M aiscetteparodieindiqueàsamanièrelaforce de la convention, puisqu'elle permet que le narrateur joue avec elle (cf. D iderot, dans J acqueslef ataliste, ou J. R oubaud, danslatrilogied'H ortense). En sorte que l'existence d'une convention s'en trouve en quelque façon validée, peut-être malgré l'écrivain...

\section{RÉFÉREN CES B I B L I O GRAPH I Q UES}

BAL, M . [1977] : N arratologie, Paris, Klincksieck.

BAn FIELD, A. [1995]: Phrases sans parole. Théorie du récit et style indirect libre, Paris, Seuil.

BERT H ON N EAU, A.M . [1993] : «Pour unenouvelleapprochedel'imparfait: l'imparfait, un tempsanaphoriqueméronomique», en coll. avec G. Kleiber, dans Langages, $n^{0} 112$, Paris, Larousse, 55-73.

Charaudeau, P. [1992]: Grammaire du sens et de l'expression, Paris, $\mathrm{H}$ achette.

COM BETTES, B. [1992]: L'O rganisation du texte, U niversité de M etz, coll. «D idactique des textes».

D AN ON -BOILEAU, L. [1980]: Énonciation et référenciation dans les textes littérairesfrançaiset anglais, tomesI et II, thèsededoctorat d'État, U niversité de Paris VII

[1982]: Produire le fictif, Paris, Klincksieck.

DEN D ALE, P. et L. T ASM OW SKI (s. la dir. de) [1994]: Les Sourcesdu savoir et leurs marques linguistiques, Langue française, $n^{0} 102$, Paris, Larousse.

FontAnILle, J. [1988]: «Point de vue: essai de définition discursive», Protée, vol. 16, no 1-2, 7-22

[1989]: LesE spacessubjectifs. Introduction à la sémi otiquedel'observateur, Paris, $\mathrm{H}$ achette université.

GenetTe, G. [1972]: Figures III, Paris, Seuil;

[1983] : N ouveau D iscours sur le récit, Paris, Seuil.

GUILLEM IN -FLESCH ER, ]. [1984] : «Énonciation, perception et traduction», Langages, n0 73, Paris, Larousse, 74-97.

KeRBRAT-O RECCHIONI, C. [1990]: LesI nteractionsverbales, tomel, Paris, Armand Colin.

LIN TVELT, J. [1981] : Essai de typologie narrative, Paris, J. Corti.

M AIN GU EN EAU, D . [1986] : Éléments de linguistique pour le textelittéraire, Paris, Bordas.

PoulLLon, J. [1946] : Temps et Roman, Paris, G allimard (rééd. [1993], Paris, Gallimard, coll. «TEL »).

RABATEL, A. [1996] : Approchesémio-linguistiquedela notion depoint devue thèse de doctorat, U niversité de $M$ etz;

[1997a] : «L'introuvablefocalisation externe», Littérature, no 107, Paris, Larousse, 88-113;

[1997b]: U nehistoiredu point devue, Paris, K lincksieck/C entred'études linguistiques des textes et des discours, U niversité de M etz;

[1998]: La Construction textuelle du point de vue, Lausanne, Paris, D elachaux et $\mathrm{N}$ ieslé;

[2000]: «U n, deux, troispointsdevue?Pour uneapprocheunifiantedes points de vue narratif et discursif $»$, La lecture littéraire, $n^{0} 4$, U niversité de Reims.

TO D O RO V, T . [1968] : Poétique(texteremaniéen 1973), dans Q u'est-ceque le structuralisme? (en coll. avec O. Ducrot, D. Sperber, M. Safouan et F. W ahl), Paris, Seuil;

[1972]: D ictionnaire encyclopédique des sciences du langage (en coll. avec 0 . D ucrot), Paris, Seuil.

VIT O UX, P. [1982]: «Le jeu de la focalisation », Poétique, n051, 359-368;

[1988]: «Focalisation, point de vue, perspective», Protée, vol. 16, n0 12, 33-38.

W EIN RICH , H . [1973] : LeT emps, trad. éd. all. [1964], Paris, Seuil. 